\title{
Clinical trials of Ebola therapies to begin in December
}

\author{
Anne Gulland
}

London

The first clinical trials of therapies for Ebola virus disease will begin next month in the treatment centres run by Médecins Sans Frontières in Guinea.

At a press conference in Geneva the principal investigators of the trials announced that three therapies would be tested at three separate sites. They said that the trials would begin in December, and 100-200 patients at each treatment centre would undergo testing.

A team led by the French National Institute of Health and Medical Research will test the antiviral drug favipiravir in Gueckedou, the antiviral brincidofovir will be tested by a consortium led by the University of Oxford at a site yet to be announced, and convalescent whole blood and plasma therapy will be tested by a group led by the Antwerp Institute of Tropical Medicine in Conakry.

The treatments were chosen from a list drawn up by the World Health Organization and were selected for their safety, efficacy, and-importantly-availability. Both favipiravir and brincidofovir have been tested in humans with influenza virus. Other experimental drugs such as ZMapp, which was given to UK nurse Will Pooley when he became infected with the virus, were not available in large enough quantities to test.

Annick Antierens, coordinator of the trials for Médecins Sans Frontières, said, "One of the major issues has been availability. Some promising drugs were not considered because there is not availability." She added that the number of trial sites may be expanded as more drugs became available.

Peter Horby, who is leading the Oxford University trial, said that there was both a humanitarian and scientific need to carry out the trials now. "We have these products which may or may not work. The only way to test them is during an epidemic. If we're going to find a treatment we have to do it now. We're going fast by clinical trials standards but maybe not fast enough for the people on the ground," he said.

He said that everyone involved in the trial had left their "comfort zone," including the institutions that were fast tracking the trials,
Médecins Sans Frontières (which had not previously been involved in a clinical trial during a disease outbreak), pharmaceutical companies, and the authorities in Guinea.

The studies will be conducted on a single arm basis, and they have a broad target of 14 day survival. The therapies will be tested on both adults and children, although pregnant women will be excluded from the trials of the antiviral drugs. If mortality drops below $40 \%$ the trials will be terminated, and if survival exceeds $80 \%$ the trial will be stopped and the drugs will be made available to other patients.

The researchers said that they expected the first results in February and March next year, but Horby said that it would depend on the number of patients who were seen at the trial sites and consented to the treatment. "The other unknown is the effect of the drug. If there's a big effect we will get the answer quicker," he said

The Dutch researchers will test whole blood products first, while the logistics of plasma production are put in place. Johan van Griensven, principal investigator on the trial, said that the most important aspect of the trial was engaging with the community. "We're conducting an in-depth anthropological evaluation to understand whether such an intervention - blood donation used for treatment - is acceptable to the community in Guinea and to Ebola survivors," he said.

The latest figures on the disease from WHO showed 14098 cases, including 5160 deaths. Data showed that the rate of the disease had increased in Sierra Leone with 1211 cases in the last 21 days compared with 466 in Liberia (which has seen the largest number of cases overall) and 325 in Guinea.

Access all of The BMS s content on the ongoing Ebola outbreak at thebmj.com/ebola.

Cite this as: BMJ 2014;349:96827

๑) BMJ Publishing Group Ltd 2014 